\title{
THE IMPORTANCE OF OMEGA-3 ESSENTIAL FATTY ACIDS IN PREGNANCY. IS THE VEGAN DIET SAFE FOR PREGNANT WOMEN?
}

\author{
LORENA EMANUELA BUTA ${ }^{1}$, AMELIA TERO-VESCAN ${ }^{2}$ \\ 1,2 “George Emil Palade” University of Medicine, Pharmacy, Science and Technology of Tîrgu Mureș
}

\begin{abstract}
Keywords: omega 3 fatty Abstract: Omega 3 fatty acids (n-3PUFA) are essential compounds in humans, their biological role acids, pregnancy, being dependent on food intake, mostly of animal origin. In the newborn, $n$-3PUFA are involved in the lactation, brain formation of membrane phospholipids in the retina and grey matter influencing vision and development neurogenesis. The purpose of this review is to study literature data regarding $n$-3PUFA intake in vegan pregnant women, with beneficial effects and supplementation possibilities. The conclusions of our study confirm the benefits of the vegan diet in pregnant women, as long as nutritional deficiencies are carefully managed through the administration of appropriate dietary supplements.
\end{abstract}

\section{INTRODUCTION}

The number of people who choose a vegan diet is increasing, so it is of particular importance to assess the safety of such a decision during pregnancy. Literature data are controversial, while The American Dietetic Association and The Academy of Nutrition and Dietetics consider a well-planned vegan diet to be adequate to maintain health at all stages of life, including pregnant or breastfeeding women, The German Nutrition Society and the European Society for Paediatric Gastroenterology, Hepatology and Nutrition do not recommend following a vegan diet during pregnancy.(1-3) Vegan diet has several disadvantages as it excludes any product of animal origin, being characterized by a lower content of saturated fatty acids, cholesterol, essential micronutrients (iron, zinc, omega-3 fatty acids, vitamin B12, vitamin D, calcium, iodine) but also advantages, a higher intake of antioxidants, dietary fibres, folic acid, phytochemicals and carotenoids.(4)

De novo biosynthesis of fatty acids in the body leads to the formation of saturated and monounsaturated fatty acids, but polyunsaturated fatty acids (PUFA), both omega 3 PUFA (n3PUFA) and omega 6 (n-6PUFA) cannot be synthesized by mammalian cells and if we refer to the vegan diet, they come in the form of two precursors, linoleic acid (LA, C18:2n-6), for the class of n-6PUFA, respectively $\alpha$-linolenic acid (ALA, C18:3n3 ), for the class n-3PUFA. $(5,6)$ These precursors are synthetized from stearic acid (C18:0) in plant cells by a combined action of $\Delta^{12}$ and $\Delta^{9}$ desaturases, enzymes that are not active in the human body. Consequently, these nutrients must be supplemented through diet. Further on, through a series of desaturations and elongations that occur mainly in the liver, these precursors are converted into arachidonic acid (AA, 20:4n-6), eicosapentaenoic acid (EPA, 20:5n-3) and docosahexaenoic acid (DHA, 22:6n$3) .(7,8) \mathrm{AA}$ is a substrate for the formation of proinflammatory eicosanoids (prostaglandins E2 and leukotrienes B4) and is involved in cellular signaling pathways while eicosanoids derived from the n-3PUFA, prostaglandins E3 and leukotrienes B5, have opposite anti-inflammatory effect.(6) Strong mediators such as protectins (derived from DHA) and resolvins (derived from DHA and EPA) are beneficial in the management of inflammation.(9) Some metabolic pathways for PUFA are shown in figure no. 1.

LA and ALA are both metabolized in the same way, so they compete for elongation and desaturating enzymes. Therefore, increased LA consumption decreases DHA synthesis from ALA, which is why it is necessary to maintain an optimal ratio of n-6 and n-3PUFA intake, in order to provide sufficient amounts of EPA and DHA. The recommended ratio for maintaining optimal body functions is between $2: 1$ and 3:1.(10)

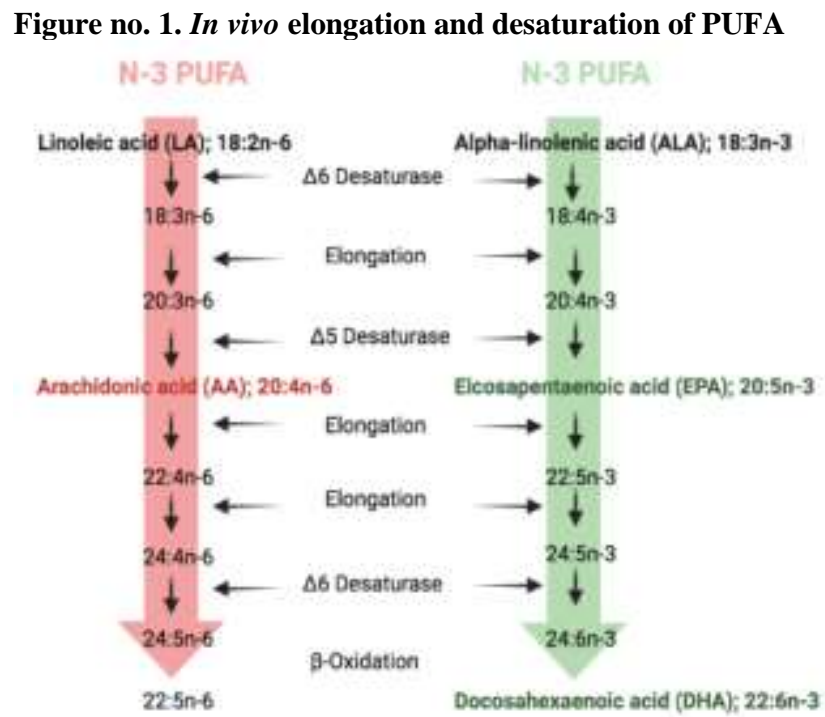

The purpose of this review is to emphasize the most important aspects related to the biochemistry of n-3PUFA, but also their role during pregnancy, on the fetus and during lactation in women who follow a vegan diet.

${ }^{2}$ Corresponding author: Amelia Tero-Vescan, Str. Gh. Marinescu, Nr. 38, Tîrgu Mureș, România, E-mail: amelia.tero-vescan@umfst.ro, Phone: $+40265215551$

Article received on 06.10.2021 and accepted for publication on 26.11.2021 


\section{CLINICAL ASPECTS}

\section{Omega-3 status in vegans}

In case of vegetarians and especially vegans, achieving a proper omega 3 status is more difficult, as their diet, rich in nuts, seeds, certain vegetables and vegetal oils, limits the sources of n-3PUFA, while providing considerable amounts of LA, the most abundant $\mathrm{n}$-6PUFA.(11,12) Literature data show for vegans and vegetarians an average daily intake of $19.4 \mathrm{~g} \mathrm{LA}$ and $1.34 \mathrm{~g}$ ALA, compared to $13.1 \mathrm{~g} \mathrm{LA}$ and $1.43 \mathrm{~g}$ ALA in omnivores.(6) Since there are few ALA-rich plant sources compared to LA, it is a challenge for vegans to acquire the daily requirements (DRI) for ALA.(13) However, important sources of ALA include: ground flax seeds, nuts, soybeans.(14) LA and ALA DRI are provided through diet and, as vegans have no source of EPA and DHA in the diet, they rely only on endogenous transformation of the precursor ALA, with a modest conversion rate in humans (5-8 \%). Important sources of EPA and DHA are found in fish, seafood, respectively seaweed, meat or eggs, where they come from endogenous conversion of ALA and are further stored in cells and adipose tissue. Therefore, given that the sources of EPA and DHA are almost exclusively of animal origin, vegans are limited to algae-based supplements to satisfy this need.(13) Most literature studies confirm these data, showing that the intake of DHA and EPA is minimal in vegans, tending to 0 , unless additional sources are added in the diet.(15-18)

Studies regarding the effects/deficiency of $n$ 3PUFA on the fetus and pregnancy

The literature data show numerous advantages of a low-fat diet but rich in fruits, vegetables and fibres. Such a diet seems to be beneficial at any stage of life. Among the benefits being a lower incidence rate of obesity, diabetes, better lipid profile, maintaining blood pressure within normal limits, cancer prevention.(4) Pregnant vegan women have a lower than average risk of cesarean delivery, neonatal and maternal mortality, gestational diabetes and excessive weight gain. In addition, the incidence of preeclampsia, directly associated with increased fat and sugar intake and low fibre, appears to be lower among vegan mothers.

Although the role of fatty acids as an energy source is well known, of particular importance is their role in intrauterine development, being components of membrane phospholipids, precursors of eicosanoids, ligands for membrane receptors and transcription factors that regulate gene expression.(7) DHA is part of membrane phospholipids in the retina and gray matter of the brain. $(7,19)$ During the last semester of pregnancy, as well as immediately after birth, DHA accumulates rapidly in the brain and retina, so its deficiency negatively influences the process of vision, neurogenesis, growth, expression of genes and synthesis of neurotransmitters, including serotonin and dopamine metabolism. $(20,21)$ DHA is also stored in the adipose tissue of the fetus in a 50 times higher amount than in the brain. After birth, these deposits have an increased turnover rate, with a specific role in maintaining brain and vision development during the first months of life.(22)

Given the biological activity of n-3PUFA, some researchers have hypothesized that their maternal levels influence pregnancy outcomes (gestational age, birth weight, postpartum depression) and fetal development (cognitive and immune function).(21,23) The results of several observational and interventional studies described in recent scientific literature are:

- Increased intake of n-3PUFA during pregnancy can lead to an increase in gestation time and birth weight, with no described side effects. Given that premature birth is the leading cause of neonatal mortality and morbidity in the United States, this can be considered a positive outcome. The postulated mechanism that explains this phenomenon is a decreased production of prostaglandins $\left(\mathrm{PGE}_{2}\right.$ and $\left.\mathrm{PGF}_{2 \alpha}\right)$ and an increased production of prostacyclin $\left(\mathrm{PGI}_{2}\right)$ consecutive to a higher EPA food intake, causing relaxation of the myometrium and delayed onset of labour. However, there are also studies that dismiss a correlation between fish oil supplementation and gestational age. $(21,24,25)$

- a decreased synthesis of maternal thromboxane $A_{2}$ and a reduced sensitivity to angiotensin II was observed following the intake of high doses of n-3PUFA.(26) However, despite promising observational data, there is insufficient evidence from randomized, placebo-controlled studies to demonstrate a significant effect on the incidence or severity of preeclampsia.(21)

- $\quad$ significant benefits on cognitive development in children were obtained by supplementing the diet of pregnant women with n-3PUFA. The greatest benefit demonstrated was on visual acuity, followed by mental development index (MDI) on Bayley Developmental Scale (BSID). No influence was observed on the average IQ during childhood, noting that the analysis included a small number of studies.(27)

- there is an inversely proportional association between the level of n-3PUFA in the umbilical cord and the development of allergic diseases during childhood (asthma, atopic dermatitis and allergic rhinitis). DHA and EPA act as a substrate for cytokines and antibodies involved in modulating allergic inflammatory responses, thus promoting the development of immune cells.(28)

- $\quad$ some studies show that increased intake of PUFA during pregnancy decreases the risk of postpartum depression by decreasing the production of proinflammatory cytokines involved. However, this conclusion is inconsistent, as randomized and controlled studies have failed to demonstrate a clear mechanism through which dietary supplementation with n-3PUFA could prevent these symptoms. $(29,30)$

Optimal intake of n-3PUFA in pregnancy and vegan alternatives

DHA accumulation begins in utero, mostly by transfer through the placenta, the highest amounts being acquired between 26-40 weeks after conception.(31) Fetal concentrations of DHA reflect the maternal diet, as the human body is not able to efficiently convert ALA to DHA.(24) Although the conversion rate is slightly higher in women and metabolic differences appear to increase the level of maternal DHA during pregnancy, the need may exceed production during this period, due to the fetal requirements of about 50-60 mg per day in the last trimester.(21) Thus, without adequate supplementation, vegan women do not appear to be able to compensate for the low intake and provide enough DHA for fetal development.(32)

Although there is no unanimous consent on a specific DRI during pregnancy and postpartum, most experts indicate 200-300 mg/day of DHA or EPA. This is also the position of the American Dietetic Association, given the importance of DHA and the low levels present in the milk of vegan mothers. Direct sources of DHA and EPA can be found in supplements derived from microalgae, but also in some prenatal vitamins.(33)

It is also important to ensure a qualitative source of DHA that eliminates the risks of contamination with heavy metals, dioxins and polychlorinated bisphenols that may pose a health threat. Thus, food supplements obtained from Schizochytrium species, using biotechnology that allows their growth under controlled conditions, are a safe source.(34)

American Dietetic Association and the Academy of Nutrition and Dietetics consider a diet that completely excludes 


\section{CLINICAL ASPECTS}

products of animal origin to be safe during pregnancy, as long as it is well planned. However, there is an increased incidence of nutrient deficiencies in vegans, including n-3PUFA, which is why, during pregnancy, when most DRI are higher, vegan women should consult a specialist to ensure proper supplementation.

n-3PUFA, especially DHA, are very important for fetal growth, especially in the development of the brain and vision. For this reason, and because prospective studies evaluating their effect on the fetus are relatively limited by ethical considerations, further research is needed to more clearly define the optimal intake in specific populations. Although there are some disadvantages, vegan diets also bring a number of important benefits.

\section{REFERENCES}

1. Craig WJ, Mangels AR. American Dietetic Association. Position of the American Dietetic Association: vegetarian diets. J Am Diet Assoc. 2009;109(7):1266-82.

2. Melina V, Craig W, Levin S. Position of the Academy of Nutrition and Dietetics: Vegetarian Diets. J Acad Nutr Diet. 2016;116(12):1970-80.

3. Richter M, Boeing H, Funk DO, et al for the German Nutrition Society (DGE). Vegan Diet. Position of the German Nutrition Society. Ernahrungs Umschau. 2016;63(4):92-102.

4. Sebastiani G, Herranz Barbero A, Borrás-Novell C, et al. The Effects of Vegetarian and Vegan Diet during Pregnancy on the Health of Mothers and Offspring. Nutrients. 2019;11(3):557.

5. Deckelbaum RJ, Calder PC, Harris WS, et al. Conclusions and recommendations from the symposium, Heart Healthy Omega-3s for Food: Stearidonic Acid (SDA) as a Sustainable Choice. J Nutr. 2012;142(3):641S-643S

6. Saunders AV, Davis BC, Garg ML. Omega-3 polyunsaturated fatty acids and vegetarian diets. Med J Aust. 2013;199(S4):S22-6

7. Innis SM. Fatty acids and early human development. Early Human Development, 2007; 83(12):761-6.

8. Innis SM. Perinatal biochemistry and physiology of longchain polyunsaturated fatty acids. J Pediatr. 2003;143(4):S1-8.

9. Kohli P, Levy BD. Resolvins and protectins: mediating solutions to inflammation. $\mathrm{Br} \mathrm{J}$ Pharmacol 2009;158(4):960-71.

10. Kris-Etherton PM, Taylor DS, Yu-Poth S, et al Polyunsaturated fatty acids in the food chain in the United States. Am J Clin Nutr. 2000;71(1):179S-88S.

11. Davis BC, Kris-Etherton PM. Achieving optimal essential fatty acid status in vegetarians: current knowledge and practical implications. Am J Clin Nutr. 2003;78(3):640S$646 \mathrm{~S}$.

12. Sanders TA. Essential fatty acid requirements of vegetarians in pregnancy, lactation, and infancy. Am J Clin Nutr. 1999;70(3):555S-9S.

13. Burns-Whitmore B, Froyen E, Heskey C, et al. AlphaLinolenic and Linoleic Fatty Acids in the Vegan Diet: Do They Require Dietary Reference Intake/Adequate Intake Special Consideration?. Nutrients. 2019;11(10):2365.

14. Penney DS, Miller KG. Nutritional counseling for vegetarians during pregnancy and lactation. J Midwifery Womens Health. 2008;53(1):37-44.

15. Sanders TA, Roshanai F. Platelet phospholipid fatty acid composition and function in vegans compared with ageand sex-matched omnivore controls. Eur J Clin Nutr. 1992;46(11):823-31.

16. Fokkema MR, Brouwer DA, Hasperhoven MB, et al. Short- term supplementation of low-dose gamma-linolenic acid (GLA), alpha-linolenic acid (ALA), or GLA plus ALA does not augment LCP omega 3 status of Dutch vegans to an appreciable extent. Prostaglandins Leukot Essent Fatty Acids. 2000;63(5):287-92.

17. Mann N, Pirotta Y, O'Connell S, et al. Fatty acid composition of habitual omnivore and vegetarian diets. Lipids. 2006;41(7):637-46.

18. Sarter B, Kelsey KS, Schwartz TA, et al. Blood docosahexaenoic acid and eicosapentaenoic acid in vegans: Associations with age and gender and effects of an algalderived omega-3 fatty acid supplement. Clin Nutr. 2015;34(2):212-8.

19. Das UN. Essential Fatty acids - a review. Curr Pharm Biotechnol. 2006;7(6):467-82.

20. Innis SM. Dietary (n-3) Fatty Acids and Brain Development, The Journal of Nutrition, 2007;137(4):8559.

21. Jensen CL. Effects of n-3 fatty acids during pregnancy and lactation. Am J Clin Nutr. 2006;83(6):1452S-7S .

22. Haggarty P. Effect of placental function on fatty acid requirements during pregnancy. Eur J Clin Nutr. 2004;58(12):1559-70.

23. Akabas SR, Deckelbaum RJ. Summary of a workshop on n-3 fatty acids: current status of recommendations and future directions. Am J Clin Nutr. 2006;83(6):1536S-8S.

24. Coletta JM, Bell SJ, Roman AS. Omega-3 Fatty acids and pregnancy. Rev Obstet Gynecol. 2010;3(4):163-71.

25. Olsen SF, Hansen HS, Sommer S, et al. Gestational age in relation to marine n-3 fatty acids in maternal erythrocytes: a study of women in the Faroe Islands and Denmark. Am J Obstet Gynecol. 1991;164(5):1203-9.

26. Adefegha SA. Functional Foods and Nutraceuticals as Dietary Intervention in Chronic Diseases; Novel Perspectives for Health Promotion and Disease Prevention. J Diet Suppl. 2018;15(6):977-1009.

27. Shulkin M, Pimpin L, Bellinger D, et al. n-3 Fatty Acid Supplementation in Mothers, Preterm Infants, and Term Infants and Childhood Psychomotor and Visual Development: A Systematic Review and Meta-Analysis. J Nutr. 2018;148(3):409-18.

28. Blümer N, Renz H. Consumption of omega3-fatty acids during perinatal life: role in immuno-modulation and allergy prevention. J Perinat Med. 2007;35(1):S12-8.

29. Makrides M, Gibson RA, McPhee AJ, et al. Effect of DHA supplementation during pregnancy on maternal depression and neurodevelopment of young children: a randomized controlled trial. JAMA. 2010;304(15):1675-83.

30. Freeman MP, Davis M, Sinha P, et al. Omega-3 fatty acids and supportive psychotherapy for perinatal depression: a randomized placebo-controlled study. J Affect Disord. 2008;110(1-2):142-8.

31. Martinat M, Rossitto M, Di Miceli M, et al. Perinatal Dietary Polyunsaturated Fatty Acids in Brain Development, Role in Neurodevelopmental Disorders. Nutrients. 2021;13(4):1185.

32. Mollica MP, Trinchese G, Cimmino F, et al. Milk Fatty Acid Profiles in Different Animal Species: Focus on the Potential Effect of Selected PUFAs on Metabolism and Brain Functions. Nutrients. 2021;13(4):1111.

33. American Dietetic Association; Dietitians of Canada. Position of the American Dietetic Association and Dietitians of Canada: Vegetarian diets. J Am Diet Assoc. 2003;103(6):748-65

34. Miedziaszczyk M, Ciabach P, Grześkowiak E, et al. The Safety of a Vegan Diet During Pregnancy. Postepy Hig Med Dosw, 2021;75:417-25. 